\title{
Correlation Functions and Spin
}

\author{
Tomáš Tyc \\ Dept. of Theor. Physics, Masaryk University, 611 37 Brno, Czech Republic \\ email: tomtyc@physics.muni.cz
}

June 2000

\begin{abstract}
The $k$-electron correlation function of a free chaotic electron beam is derived with the spin degree of freedom taken into account. It is shown that it can be expressed with the help of correlation functions for a polarized electron beam of all orders up to $k$ and the degree of spin polarization. The form of the correlation function suggests that if the electron beam is not highly polarized, observing multi-particle correlations should be difficult. The result can be applied also to chaotic photon beams, the degree of spin polarization being replaced by the degree of polarization.
\end{abstract}

PACS numbers: 05.30.Fk, 25.75.Gz

\section{Introduction}

Although the theory of multi-particle correlations in free-electron beams is relatively well developed (see e.g. [1, 2, 3, 4, 5, 61), up to now there has not been much attention devoted to the electron spin. We feel this as a deficiency because the spin degree of freedom is a significant feature of the electron and should be therefore taken into account. In this paper we try to reduce this deficiency for one particular case that is, as we believe, the most typical one in electron correlation experiments. Namely, we calculate the multi-electron correlation function for an electron chaotic beam with an arbitrary degree of spin polarization.

Moreover, the presented theory can be applied not only to chaotic electron (or generally spin1/2-particle) beams, but also to a chaotic photon beams because the corresponding Hilbert space of possible photon polarization states is two-dimensional precisely as is the Hilbert space of the spin- $1 / 2$ states. We will speak about electrons for brevity, but the argumentation and the results can be applied also to the case of photons.

\section{Chaotic state}

The reason why we concentrate on the chaotic state is that we believe it is a good approximation for a state produced by the most coherent electron source available nowadays - the field-emission tip. This source is the only candidate for electron correlation experiments at the present time [2] because it offers both high brightness and a relatively monochromatic energy spectrum. The electrons emitted from a field-emission tip originate from a quasi-equilibrium state in the metal very close to a thermal state, which is an example of the chaotic state. We do not expect an additional coherence to come into existence during the tunneling process and therefore suppose that the state remains chaotic also outside the metal!.

The chaotic state has been first introduced by Glauber for a mode of the electromagnetic field [8] as a state with a maximum entropy for a given occupation number of this mode. We generalize this definition to an electron field with the spin taken into account: the chaotic state is a state of a maximum entropy for given occupation numbers of the individual modes of the field and for a given spin polarization.

\footnotetext{
${ }^{1}$ this might be no longer true for a polarized field-emission source [7], where a correlation between the spin and coordinate can come into existence; however, it can be shown that our results remain valid as long as the energy spectra of the spin components "up" and "down" are close to each other
} 
From the condition of the maximum entropy it follows that there is no correlation between the coordinate and the spin components of the chaotic state, which allows to treat the spin degree of freedom separately from the coordinate degrees of freedom. In calculating the correlation functions one can avoid in this way the formalism of spin-dependent electron field operators and use a relatively simple argumentation based on the probability theory. As will be seen in the following, this enables to express the correlation functions of a partially spin-polarized chaotic electron beam in terms of the degree of spin polarization and the correlation functions corresponding to a completely polarized beam, the form of which is known [4, 6 .

For our purpose it is fully sufficient to define the $k$-electron correlation function $O^{(k)}\left(\vec{r}_{1}, t_{1}, \ldots, \vec{r}_{k}, t_{k}\right)$ as the probability of detecting $k$ electrons at the $k$ space-time points $\left(\vec{r}_{1}, t_{1}\right),\left(\vec{r}_{2}, t_{2}\right), \ldots,\left(\vec{r}_{k}, t_{k}\right)$. To get a more compact form of the equations, the shortened notation $O_{12 \ldots k}^{(k)}$ instead of $O^{(k)}\left(\vec{r}_{1}, t_{1}, \ldots, \vec{r}_{k}, t_{k}\right)$ will be used, each index $i$ standing for one space-time point $\left(\vec{r}_{i}, t_{i}\right)$. Analogous correlation functions for a spin-polarized beam will be denoted by $G$ instead of $O$, so for example the two-electron correlation function for a polarized beam is $G_{1.2}^{(2)}$. For a more precise definition of correlation functions in terms of electron field operators see e.g. [1], 6].

\section{Two spin-polarized sources}

Consider an electron that has been emitted from the source. Its state can be described by the spin density operator $\hat{\rho}_{s}$ that is represented by a Hermitian matrix of the second order in any orthonormal basis. As $\hat{\rho}_{s}$ can be diagonalized by a unitary transformation, there exists an orthonormal basis $\{|1\rangle,|2\rangle\}$ in which $\hat{\rho}_{s}$ has the diagonal form

$$
\hat{\rho}_{s}=\left(\begin{array}{cc}
\rho_{1} & 0 \\
0 & \rho_{2}
\end{array}\right)=\rho_{1}|1\rangle\left\langle 1\left|+\rho_{2}\right| 2\right\rangle\langle 2|
$$

The states $|1\rangle,|2\rangle$ correspond to the spin orientation "up" and "down", respectively, with respect to some particular axis $\vec{a}$ in space. In the case of photons, the states $|1\rangle,|2\rangle$ would describe two orthogonal polarizations, e.g. two linearly polarized waves with the polarization planes perpendicular to each other or a pair of the left- and right circularly polarized waves, depending on the properties of the source.

It is useful to express the probabilities $\rho_{1}$ and $\rho_{2}$ in terms of the degree of polarization. This quantity is defined as $P=\left(\rho_{1}-\rho_{2}\right) /\left(\rho_{1}+\rho_{2}\right)=\rho_{1}-\rho_{2}$ (provided that $\left.\rho_{1} \geq \rho_{2}\right)$, which yields

$$
\rho_{1}=\frac{1+P}{2}, \quad \rho_{2}=\frac{1-P}{2} .
$$

Now, the spin state of the ensemble of electrons coming from the source is completely described by the density operator (1). As can be seen from its form, $\hat{\rho}_{s}$ corresponds to the situation as if just two types of electrons were emitted from the source: first, electrons polarized up with respect to the axis $\vec{a}$ and second, electrons polarized down with respect to $\vec{a}$. The probabilities that an electron emitted from the source is of the first or second type are $\rho_{1}$ or $\rho_{2}$, respectively. The fact that the spin density operator $\hat{\rho}_{s}$ is diagonal means that there is no correlation between the two spin components up and down. At the same time, there is no correlation between the spin and the coordinate because the complete multi-electron state is chaotic. This allows the following consideration to be made.

We substitute formally the original electron source $S$ by two independent sources $S_{\text {up }}$ and $S_{\text {down }}$ that emit electrons polarized up and down with respect to the axis $\vec{a}$, respectively. If this should be correct, the sources $S_{\text {up }}$ and $S_{\text {down }}$ must have the same properties (of course except for the spin) as the original source $S$ has. This implies for example that they are located at the place of the original source $S$ and have the same energy spectrum. Moreover, the emission intensities of the sources $S_{\text {up }}$ and $S_{\text {down }}$ must be equal to $\rho_{1}$ and $\rho_{2}$ times the intensity of the original source $S$, respectively. In the following, the idea of substituting formally the original source by two polarized ones will be used for a direct derivation of the correlation function for a partially polarized electron beam. To see the idea more clearly, we will consider the simple case $k=2$ first and then go over to a general $k$. 


\section{Two-electron correlation function}

The two-electron correlation function expresses the probability of the event of detecting two electrons at the space-time points $\left(\vec{r}_{1}, t_{1}\right)$ and $\left(\vec{r}_{2}, t_{2}\right)$. This event can happen in one of four ways that are distinguishable in principle because the coordinate and spin operators mutually commute:

1. the spins of both electrons are oriented up with respect to the axis $\vec{a}$,

2. the spins of both electrons are oriented down,

3. the spin of the electron at $\left(\vec{r}_{1}, t_{1}\right)$ is oriented down, the spin of the electron at $\left(\vec{r}_{2}, t_{2}\right)$ is oriented up,

4. the spin of the electron at $\left(\vec{r}_{1}, t_{1}\right)$ is oriented up, the spin of the electron at $\left(\vec{r}_{2}, t_{2}\right)$ is oriented down.

If cases 1 or 2 occur, then according to the previous section we deal with two electrons from the same polarized source. Therefore the two-electron correlation function is equal to the analogous correlation function $G_{12}^{(2)}$ for polarized electrons. On the other hand, if cases 3 or 4 occur, we deal with two electrons from two independent, oppositely polarized sources. The electrons are then completely uncorrelated and the correlation function is equal to the product of one-electron correlation functions, i.e., $G_{1}^{(1)} G_{2}^{(1)}$. As the probability that one electron is polarized up or down is $\rho_{1}$ or $\rho_{2}$, respectively, the probabilities of the cases 1.-4. are $\rho_{1}^{2}, \rho_{2}^{2}, \rho_{1} \rho_{2}$, and $\rho_{1} \rho_{2}$, respectively. The total correlation function $O_{12}^{(2)}$ can be then written as the weighed average of the polarized correlation functions,

$$
O_{1,2}^{(2)}=\left(\rho_{1}^{2}+\rho_{2}^{2}\right) G_{1,2}^{(2)}+2 \rho_{1} \rho_{2} G_{1}^{(1)} G_{2}^{(1)},
$$

which is the desired result for $k=2$.

\section{$5 \quad k$-electron correlation function}

In the derivation of the $k$-electron correlation function for a partially polarized chaotic electron beam we proceed in a completely analogous way. If $k$ electrons at the space-time points $\left(\vec{r}_{1}, t_{1}\right), \ldots,\left(\vec{r}_{k}, t_{k}\right)$ should be detected, there are $2^{k}$ possibilities how they can be polarized (instead of the four possibilities discussed in the previous section). We denote each of them by the sequence $s_{1}, s_{2}, \ldots, s_{k}$, every $s_{i}$ expressing the spin polarization of the electron at the point $\left(\vec{r}_{i}, t_{i}\right)$ and having one of two possible values, 1 for spin up and or 2 for spin down. The probability $P\left(s_{1}, \ldots, s_{k}\right)$ that the electrons have polarizations $s_{1}, \ldots, s_{k}$ is equal to $\rho_{1}^{n_{1}} \rho_{2}^{n_{2}}$, where $n_{1}$ and $n_{2}$ expresses how many times there appears 1 and 2 among the numbers $s_{1}, \ldots, s_{k}$, respectively. If $O_{1, \ldots, k}^{(k)}\left(s_{1}, \ldots, s_{k}\right)$ denotes the $k$-electron correlation function for this particular spin combination, the total $k$-electron correlation function can be written as

$$
O_{1, \ldots, k}^{(k)}=\sum_{s_{1}, \ldots, s_{k}} P\left(s_{1}, \ldots, s_{k}\right) O_{1, \ldots, k}^{(k)}\left(s_{1}, \ldots, s_{k}\right)
$$

the sum being made over all the possibilities $s_{1}, \ldots, s_{k}$. Now, if the spin polarizations of the electrons are $s_{1}, \ldots, s_{k}$, the situation is the same as if we dealt with two independent sets of electrons - one set of $n_{1}$ up-polarized electrons originating from the source $S_{\mathrm{up}}$ and another set of $n_{2}$ down-polarized

electrons originating from the source $S_{\text {down }}$. The correlation function $O_{1, \ldots, k}^{(k)}\left(s_{1}, \ldots, s_{k}\right)$ factorizes therefore into a product of two correlation functions for polarized electrons:

$$
O_{1, \ldots, k}^{(k)}\left(s_{1}, \ldots, s_{k}\right)=G^{\left(n_{1}\right)}\left(\{\vec{r}, t\}_{\text {up }}\right) G^{\left(n_{2}\right)}\left(\{\vec{r}, t\}_{\text {down }}\right) .
$$


Here $\{\vec{r}, t\}_{\text {up }}$ and $\{\vec{r}, t\}_{\text {down }}$ denote the sets of points at which the electrons are polarized up and down, respectively. Substituting Eq. (5) into Eq. (4) and re-arranging the sum, we can write the correlation function $O_{1, \ldots k}^{(k)}$ as follows:

$$
\begin{aligned}
O_{1, \ldots, k}^{(k)}=\left(\rho_{1}^{k}\right. & \left.+\rho_{2}^{k}\right) G_{1, \ldots, k}^{(k)} \\
& +\left(\rho_{1}^{k-1} \rho_{2}+\rho_{2}^{k-1} \rho_{1}\right)\left(G_{2, \ldots, k}^{(k-1)} G_{1}^{(1)}+G_{1,3, \ldots, k}^{(k-1)} G_{2}^{(1)}+\cdots+G_{1, \ldots, k-1}^{(k-1)} G_{k}^{(1)}\right) \\
& \quad+\left(\rho_{1}^{k-2} \rho_{2}^{2}+\rho_{2}^{k-2} \rho_{1}^{2}\right)\left(G_{3, \ldots, k}^{(k-2)} G_{1,2}^{(2)}+G_{2,4, \ldots, k}^{(k-2)} G_{1,3}^{(2)} \cdots+G_{1, \ldots, k-2}^{(k-2)} G_{k-1, k}^{(2)}\right)+\ldots
\end{aligned}
$$

To see the structure of such a series better, we write down the three- and four-electron correlation functions for illustration:

$$
\begin{gathered}
O_{1,2,3}^{(3)}=\left(\rho_{1}^{3}+\rho_{2}^{3}\right) G_{1,2,3}^{(3)}+\left(\rho_{1}^{2} \rho_{2}+\rho_{2}^{2} \rho_{1}\right)\left(G_{1,2}^{(2)} G_{3}^{(1)}+G_{1,3}^{(2)} G_{2}^{(1)}+G_{2,3}^{(2)} G_{1}^{(1)}\right) \\
O_{1,2,3,4}^{(4)}=\left(\rho_{1}^{4}+\rho_{2}^{4}\right) G_{1,2,3,4}^{(4)} \\
+\left(\rho_{1}^{3} \rho_{2}+\rho_{2}^{3} \rho_{1}\right)\left(G_{1,2,3}^{(3)} G_{4}^{(1)}+G_{1,2,4}^{(3)} G_{3}^{(1)}+G_{1,3,4}^{(3)} G_{2}^{(1)}+G_{2,3,4}^{(3)} G_{1}^{(1)}\right) \\
+2 \rho_{1}^{2} \rho_{2}^{2}\left(G_{1,2}^{(2)} G_{3,4}^{(2)}+G_{1,3}^{(2)} G_{2,4}^{(2)}+G_{1,4}^{(2)} G_{2,3}^{(2)}\right) .
\end{gathered}
$$

In this way the $k$-electron correlation function for partially polarized electrons is expressed in terms of the one-, two- etc. up to the $k$-electron correlation functions for polarized electrons and the degree of polarization [that is connected with $\rho_{1}, \rho_{2}$ via the relations (2)].

The considerations made in the sections 3. - 5. as well as the results (3) and (6) can be applied step by step also to a chaotic photon field because up to now we have not supposed anything about the quantum statistics of the electrons. In fact, this statistics is hidden in the spin-polarized correlation

functions $G$ and in this way it is reflected also in the correlation function $O_{1, \ldots, k}^{(k)}$. As has been mentioned, the similarity between electrons and photons in this sense comes from the same dimensions of the photon polarization Hilbert space and the electron spin Hilbert space.

We return to the electrons again. According to [4, 6], the $k$-electron correlation function for a spin-polarized chaotic electron beam can be expressed as

$$
G_{1, \ldots, k}^{(k)}=G_{1}^{(1)} G_{2}^{(1)} \cdots G_{k}^{(1)} \operatorname{det} \hat{\gamma}
$$

where $\hat{\gamma}=\left(\gamma_{i j}\right)$ is a matrix composed of the complex degrees of coherence $\gamma_{i j}$ at the points $\left(\vec{r}_{i}, t_{i}\right)$ and $\left(\vec{r}_{j}, t_{j}\right)$. Combining Eqs. (6) and (9), we arrive at the explicit form for the correlation function for a chaotic electron beam with an arbitrary spin polarization.

\section{Influence of polarization on multi-electron correlations}

To see how the spin polarization influences the correlations in an electron beam, we first return to the case of $k=2$. According to Eq. (9), the two-electron correlation function for a spin-polarized chaotic electron beam is equal to

$$
G_{1,2}^{(2)}=G_{1}^{(1)} G_{2}^{(1)}\left(1-\left|\gamma_{12}\right|^{2}\right)
$$

where we used the fact that $\gamma_{11}=\gamma_{22}=1$. With the help of Eqs. (2) and (3), we then get for $O_{1,2}^{(2)}$

$$
O_{1,2}^{(2)}=G_{1}^{(1)} G_{2}^{(1)}\left(1-\frac{1+P^{2}}{2}\left|\gamma_{12}\right|^{2}\right)
$$


If there were no correlation between the detection probabilities at the points $\left(\vec{r}_{1}, t_{1}\right)$ and $\left(\vec{r}_{2}, t_{2}\right)$, the correlation function $O_{1,2}^{(2)}$ would be simply equal to the product of the one-electron correlation functions $O_{1}^{(1)}=G_{1}^{(1)}$ and $O_{2}^{(1)}=G_{2}^{(1)}$. Therefore the second term in the parentheses in Eq. (11) is responsible for the two-electron correlation. As we can see, this term increases with the increasing degree of polarization of the beam, varying between one half for an unpolarized beam and unity for a completely polarized beam. For electrons, this result has been known [1] but it has been derived heuristically only until now. For photons a similar effect of the polarization on the correlation function is known 9].

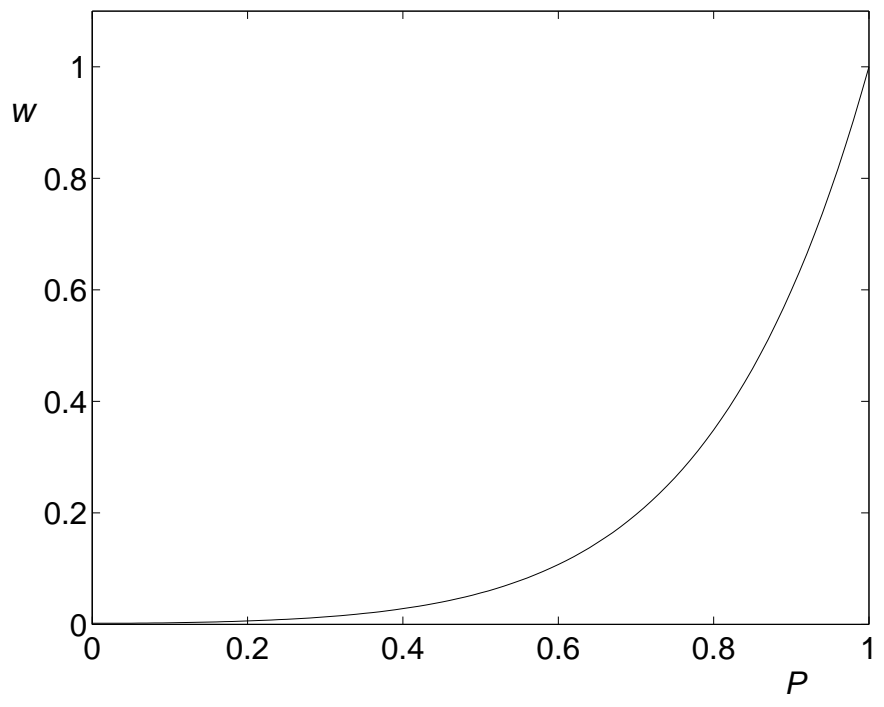

Figure 1: The factor $w=\rho_{1}^{k}+\rho_{2}^{k}=[(1+P) / 2]^{k}+[(1-P) / 2]^{k}$ expressing the intensity of the $k$-electron correlation as a function of the degree of polarization $P$ for $k=10$. As the figure shows, if $w$ should be comparable to unity, making thus the $k$-electron correlations observable, one needs a beam of a relatively high degree of polarization.

Next we go over to the case of an arbitrary $k$. According to Eq. (6), the factor $w=\left(\rho_{1}^{k}+\rho_{2}^{k}\right)$ expresses the weight of the $k$-electron correlation in the partially polarized beam compared to a completely polarized beam because the $k$-electron correlation is given just by the correlation function $G_{1,2, \ldots, k}^{(k)}$. It is evident that for large $k$ the factor $w$ is small as soon as $P$ differs even slightly from unity. Figure 1 shows the dependence $w(P)$ for $k=10$. For example if $P=0.7$, then $w$ is equal to only about 0.2 , so the ten-electron correlation is reduced to one fifth with respect to a polarized beam. Thus we must conclude that if no beam with a high degree of polarization is available, it is difficult to observe correlations of higher orders. On the other hand, from the experimental point of view observing even two-electron correlations is very difficult [2, 10]. In comparison to the extreme difficulty of, say, a ten-electron correlation experiment, making a 99\%-polarized electron beam seems to be an easy task and in this way the spin degree of freedom should have no limitation effect on the measurement of multi-electron correlations.

\section{Acknowledgments}

I would like to thank professor M. Lenc for helpful discussions. This work was supported by the Czech Ministry of Education, contract No. 144310006.

\section{References}


[1] M. Silverman, Il Nuovo Cimento 97B, 200 (1987).

[2] M. Silverman, Phys. Lett. A 120, 442 (1987).

[3] K. Toyoshima and T. Endo, Phys. Lett. A 152, 141 (1991).

[4] S. Saito et al., Phys. Lett. A 162, 442 (1992).

[5] M. Ban, Phys. Lett. A 172, 337 (1993).

[6] T. Tyc, Phys. Rev. A 58, 4967 (1998).

[7] E. Kisker, G. Baum, A. H. Mahan, W. Raith, and B. Reihl, Phys. Rev. B 18, 2256 (1978).

[8] R. Glauber: Quantum theory of coherence, in: Quantum optics, eds. S. Kay and A. Maitland, Academic Press, New York 1970.

[9] L. Mandel and E. Wolf: Optical Coherence and Quantum Optics, Cambridge University Press, 1995.

[10] T. Kodama et al., Phys. Rev. A 57, 2781 (1998).

[11] M. Silverman, Phys. Lett. A 120, 442 (1987). 